\title{
Trabalho em equipe na Neurologia: Percepções de profissionais
}

\author{
Teamwork in Neurology: Perceptions of professionals \\ Trabajo en equipo en neurología: Percepciones de los profesionales
}

Recebido: 09/07/2021 | Revisado: 14/07/2021 | Aceito: 16/07/2021 | Publicado: 25/07/2021

Pedro César Condeles

ORCID: https://orcid.org/0000-0001-6832-638X

Universidade Federal do Triângulo Mineiro, Brasil E-mail: pedrocondeles@yahoo.com.br

Lharissa Cristina Mateus

ORCID: https://orcid.org/0000-0001-9202-4218 Universidade Federal do Triângulo Mineiro, Brasil E-mail: lharissacristina@gmail.com

Letícia Gabriela de Almeida Noce ORCID: https://orcid.org/ 0000-0003-2156-6938

Universidade Federal de Uberlândia, Brasil E-mail: almeida-lg@ hotmail.com Carolina Feliciana Bracarense

ORCID: https://orcid.org/0000-0002-2363-8205 Prefeitura Municipal de Uberaba, Brasil E-mail: carolinafbracarense@gmail.com

Jéssica Fernanda Marcelina Fernandes Ferreira ORCID: https://orcid.org/0000-0003-4382-941X Universidade Federal do Triângulo Mineiro, Brasil E-mail: jefmff@gmail.com

Bibiane Dias Miranda Parreira

ORCID: https://orcid.org/0000-0001-7369-5745 Universidade Federal do Triângulo Mineiro, Brasil E-mail: bibianedias@yahoo.com.br

Bethania Ferreira Goulart

ORCID: https://orcid.org/0000-0003-2855-6767

Universidade Federal do Triângulo Mineiro, Brasil E-mail: bethaniagoulart@yahoo.com.br

\begin{abstract}
Resumo
Objetivo: identificar as percepções do trabalho em equipe segundo os profissionais de uma Unidade de Neurologia. Métodos: estudo exploratório, de abordagem qualitativa. Participaram 44 profissionais de saúde da unidade de Neurologia de um hospital público de ensino. A coleta de dados ocorreu por meio de entrevistas semiestruturadas, audiogravadas, de agosto a novembro de 2017, considerando-se a questão de pesquisa: O que pensam os profissionais da Neurologia e como vivenciam o trabalho em equipe naquele cenário? Análise de dados seguiu a Análise de Conteúdo, modalidade temática, e pautou-se no referencial teórico de processo de trabalho em saúde. Resultados: emergiram três categorias temáticas: Agentes do processo de trabalho; Instrumentos não materiais do processo de trabalho na Neurologia; e Gerência: coordenação e liderança. As categorias temáticas revelaram as percepções dos profissionais quanto ao trabalho em equipe, bem como seus facilitadores e dificultadores, na unidade de neurologia. Considerações Finais: relações interprofissionais fundamentadas na complementaridade, troca de saberes, articulação efetiva, e liderança facilitam o trabalho em equipe e contribuem para a qualidade da assistência à saúde.
\end{abstract}

Palavras-chave: Equipe de assistência ao paciente; Comportamento cooperativo; Relações interprofissionais; Cuidados críticos; Neurologia.

\footnotetext{
Abstract

Objective: to identify the perceptions of teamwork according to professionals in a Neurology Unit. Methods: exploratory study with a qualitative approach. 44 health professionals from the Neurology unit of a public teaching hospital participated. Data collection took place through semi-structured interviews, audio-recorded, from August to November 2017, considering the research question: What do Neurology professionals think and how do they experience teamwork in that scenario? Data analysis followed Content Analysis, thematic modality, and was based on the theoretical framework of the health work process. Results: three thematic categories emerged: Work process agents; Non-material instruments of the work process in Neurology; and Management: coordination and leadership. The thematic categories revealed the professionals' perceptions about teamwork, as well as its facilitators and obstacles, in the neurology unit. Final Considerations: interprofessional relationships based on complementarity,
} 
knowledge exchange, effective articulation, and leadership facilitate teamwork and contribute to the quality of health care.

Keywords: Patient care team; Cooperative behavior; Interprofessional relations; Critical care; Neurology.

\section{Resumen}

Objetivo: identificar las percepciones del trabajo en equipo según los profesionales de una Unidad de Neurología. Métodos: estudio exploratorio con abordaje cualitativo. Participaron 44 profesionales de la salud de la unidad de Neurología de un hospital universitario público. La recolección de datos se realizó a través de entrevistas semiestructuradas, grabadas en audio, de agosto a noviembre de 2017, considerando la pregunta de investigación: ¿Qué piensan los profesionales de la Neurología y cómo viven el trabajo en equipo en ese escenario? El análisis de datos siguió la modalidad de Análisis de Contenido, temática, y se basó en el marco teórico del proceso de trabajo en salud. Resultados: surgieron tres categorías temáticas: agentes del proceso de trabajo; Instrumentos no materiales del proceso de trabajo en Neurología; y Gestión: coordinación y liderazgo. Las categorías temáticas revelaron las percepciones de los profesionales sobre el trabajo en equipo, así como sus facilitadores y obstáculos, en la unidad de neurología. Consideraciones finales: las relaciones interprofesionales basadas en la complementariedad, el intercambio de conocimientos, la articulación efectiva y el liderazgo facilitan el trabajo en equipo y contribuyen a la calidad de la atención de salud.

Palabras clave: Grupo de atención al paciente; Conducta cooperativa; Relaciones interprofesionales; Cuidados críticos; Neurología.

\section{Introdução}

Os hospitais são instituições complexas e com densidade tecnológica específica. Preconiza-se que a organização do trabalho em saúde ocorra com base em equipe interprofissional, a qual viabilize a integralidade da assistência por meio de conjuntos de saberes, tecnologias e recursos necessários (Bragadóttir et al., 2017).

Trabalho em equipe interprofissional representa uma modalidade de trabalho coletivo que requer, de um lado, articulação das ações das diversas áreas profissionais, reconhecendo-se sua interdependência, e de outro, complementaridade entre agir instrumental e agir comunicativo (Peduzzi et al., 2020). Além disso, favorece o aumento do escopo de atuação dos profissionais dentro da equipe, fundamental na prática especializada, proporcionando a qualidade da atenção à saúde, segurança e satisfação tanto dos pacientes quanto dos profissionais (Peduzzi et al., 2019). Acredita-se que o trabalho em equipe represente uma potente estratégia para viabilizar trabalho coletivo e integrado, com vistas à assistência ampliada, superação da justaposição de procedimentos e ações desconexas com ênfase no adoecimento.

Na perspectiva de trabalho coletivo, apesar da proposta de trabalho em equipe, o setor saúde ainda é marcado pelo predomínio de trabalho fragmentado e parcelar dos agentes. Essa dinâmica de trabalho gera ruídos entre os profissionais e reforça a rigidez nas divisões de tarefas/ações, o que culmina no distanciamento dos integrantes da equipe de saúde e dificulta a integralidade da assistência (Rosen et al., 2018; Golom \& Schreck, 2018).

A Neurologia é uma unidade na qual os profissionais lidam cotidianamente com pacientes graves, angústia dos familiares e tênue limite entre vida e morte (Arruda et al., 2019). Isso repercute em estresse, desgaste, sobrecarga e sofrimento da equipe, interferindo nas relações interprofissionais (Purvis \& Saylor, 2019). e impactando no cuidado ofertado.

Constatam-se lacunas na produção científica a respeito de trabalho em equipe no cenário da presente investigação. Os profissionais têm capacidade técnica e habilidades para atendimento aos pacientes neurológicos. Porém, a interação profissional não articulada leva ao distanciamento entre os membros da equipe, o que prejudica o processo de tomada de decisão. Dar visibilidade às percepções dos profissionais da Neurologia sobre o seu trabalho e a perspectiva do trabalho coletivo possibilitarão trazer à tona elementos para que a gestão dispare e/ou potencialize o trabalho integrado no contexto supracitado. Diante disto, questiona-se: "o que pensam os profissionais da Neurologia e como vivenciam o trabalho em equipe naquele cenário?" O presente estudo objetivou identificar as percepções do trabalho em equipe segundo os profissionais de uma Unidade de Neurologia. 


\section{Metodologia}

Trata-se de estudo exploratório, com abordagem qualitativa, adotando-se os critérios do Consolidated criteria for reporting qualitative research (COREQ). O instrumento COREQ contempla os três domínios: equipe de pesquisa e reflexividade, desenho do estudo e análise/resultados. Tem como propósito direcionar aspectos importantes da pesquisa, garantindo a confiabilidade. A abordagem supracitada permite a compreensão das situações por meio de comportamentos empíricos, captando a essência do que está sendo investigado, não dependendo da frequência de aparição, mas sim da sua relevância (Tong et al., 2007).

Adotou-se, como referencial teórico, o processo de trabalho em saúde (Gonçalves, 1992). Isto possibilitou o diálogo entre os resultados quanto ao trabalho em equipe e os elementos do processo de trabalho, na perspectiva de integrar os pressupostos teóricos com a vivência dos agentes de uma unidade de Neurologia e contribuir para disparar discussões que favoreçam transformar os objetos de trabalho e o próprio trabalhador.

O processo de trabalho contempla os elementos: objeto de trabalho; instrumentos; finalidade e agentes. Objeto representa o que será modificado pelo trabalhador. Na presente investigação, o objeto refere-se ao indivíduo e suas condições de saúde. Para que ocorra transformação do objeto, os agentes (profissionais de saúde) utilizam instrumentos materiais, como equipamentos, medicamentos, dentre outros, e imateriais, os quais compreendem conhecimentos e saberes, com o propósito de alcançar a finalidade definida a priori, ou seja, a integralidade da assistência (Gonçalves, 1992).

A unidade de Neurologia, foco da presente pesquisa, possui 13 leitos e integra um hospital público, de ensino, de nível terciário, o qual contempla 302 leitos, em um município do interior de Minas Gerais, referência para o atendimento de alta densidade tecnológica. A referida unidade, no período da coleta dos dados, contava com 56 profissionais de saúde ligados diretamente à assistência, sendo 24 auxiliares/técnicos de enfermagem, 16 médicos, nove enfermeiros, três fisioterapeutas, um psicólogo, um fonoaudiólogo, um assistente social e um nutricionista.

Do total de 56 profissionais, foram entrevistados 44, por escolha intencional, dos quais 19 eram auxiliares/técnicos de enfermagem (43,18\%), 12 médicos (27,27\%), oito enfermeiros (18,18\%), três fisioterapeutas (6,81\%) um fonoaudiólogo $(2,28 \%)$ e um assistente social (2,28\%). Participaram aqueles que atenderam ao critério de inclusão: atuar na referida unidade há, pelo menos, um ano. Foram excluídos os que estavam afastados do trabalho durante a coleta dos dados, os que se recusaram a participar e aqueles não localizados após três tentativas para o agendamento da entrevista.

Dos 12 profissionais que não participaram, cinco não foram localizados após três tentativas para o agendamento da entrevista, cinco se recusaram a participar e dois estavam afastados do trabalho no período da coleta de dados.

A coleta de dados ocorreu de agosto a novembro de 2017, utilizando-se entrevista semiestruturada, realizada por um graduando de Enfermagem, o qual foi devidamente treinado e capacitado pela coordenadora da pesquisa. O entrevistador apresentava-se ao profissional convidado a participar, face a face, expunha o projeto, a justificativa para a sua realização e a técnica da entrevista. A entrevista foi guiada por um roteiro, elaborado pelos próprios pesquisadores, o qual foi submetido à validação aparente e de conteúdo por três doutores na temática e/ou na metodologia de pesquisa adotada. O roteiro contemplava duas partes: a primeira relacionada aos dados sociodemográficos e profissionais dos participantes; a segunda parte era composta pelas questões norteadoras para investigar, na perspectiva do profissional, como o trabalho era realizado e quais eram as suas percepções a respeito do trabalho em equipe naquela unidade.

Realizou-se teste piloto com profissionais de outro setor do hospital antes da coleta definitiva dos dados. As entrevistas foram realizadas face a face, áudio gravadas durante o horário do plantão, em momento escolhido pelo participante, em sala privativa na unidade de Neurologia, contando somente com o entrevistador e o participante e tiveram duração média de seis minutos e 30 segundos. Os participantes do estudo foram identificados como E1, E2, E3, assim por diante, até E44. 
As entrevistas foram transcritas na íntegra pelo próprio pesquisador/entrevistador. Não houve analistas externos. Para a análise dos dados, fundamentou-se na orientação metodológica da Análise de Conteúdo, modalidade Temática, seguindo-se as três etapas propostas: pré-análise, procedeu-se à leitura exaustiva do material para possibilitar a apreensão do todo dos dados e as particularidades do conjunto; exploração do material, realizou-se a sua exploração, buscando-se identificar as categorias e proceder ao agrupamento das unidades de contexto, por afinidade de conteúdo; síntese interpretativa, foi feito o agrupamento dos trechos de acordo com os temas que emergiram das entrevistas e a sua organização em unidades temáticas (Minayo et al., 2016). Os dados foram analisados à luz de uma aproximação com o referencial teórico de Processo de Trabalho (Gonçalves, 1992) e do objeto do estudo.

Da análise dos dados, emergiram três categorias temáticas: Agentes do processo de trabalho; Instrumentos não materiais do processo de trabalho na Neurologia; e Gerência: coordenação e liderança.

Esta pesquisa integra um projeto maior intitulado "Trabalho em equipe em unidades hospitalares: facilidades e dificuldades". Em conformidade com a Resolução no 466/2012, do Conselho Nacional de Saúde, o projeto foi aprovado pelo Comitê de Ética em Pesquisa envolvendo seres humanos (CEP) de uma Universidade Federal, no interior de Minas Gerais, via Plataforma Brasil. Os participantes da pesquisa assinaram o Termo de Consentimento Livre e Esclarecido (TCLE) e foram garantidos o sigilo e a privacidade.

\section{Resultados}

Dentre os 44 participantes da pesquisa, 27 eram do sexo feminino (61,36\%) e 17 do sexo masculino $(38,64 \%)$, com idades entre 24 e 65 anos, sendo a média de 37 anos. Quanto à formação, 30 (68,18\%) profissionais possuíam o ensino superior completo e $14(31,82 \%)$ ensino médio completo. Destaca-se que 19 profissionais $(43,18 \%)$ tinham algum tipo de formação complementar, dos quais 15 (78,96\%) possuíam especialização; dois (10,52\%) fizeram mestrado; um $(5,26 \%)$ especialização e mestrado; e um $(5,26 \%)$ especialização, mestrado e doutorado. O tempo médio de formação foi de 11 anos, com tempo de atuação na unidade de neurologia variando de um a 32 anos, com média de três anos e 8 meses.

Por meio da análise das entrevistas, emergiram três categorias temáticas: Agentes do processo de trabalho; Instrumentos não materiais do processo de trabalho na Neurologia; e Gerência: coordenação e liderança. As referidas categorias revelaram as percepções dos profissionais quanto ao trabalho em equipe, bem como seus facilitadores e dificultadores, na unidade de Neurologia. Destaca-se que as categorias não contemplaram subcategorias. Durante o processo de compilação das categorias, os achados emergentes na análise foram agrupados por afinidade de conteúdo nesses três eixos temáticos, os quais compreendiam as temáticas reveladas.

$\mathrm{Na}$ categoria temática Agentes do processo de trabalho, os participantes revelaram que os agentes do processo de trabalho, na Neurologia, influenciam diretamente na realização e condução do trabalho em equipe. Evidenciaram que a composição incompleta da equipe dificulta a concretização do trabalho em equipe, compromete a articulação entre as ações/condutas, fragiliza a integralidade do cuidado e prejudica a assistência ao paciente. Isto é ilustrado nas falas que seguem:

[...] às vezes falta profissional, então fica deficitária algumas coisas, esse é o principal problema, falta gente para trabalhar [...] paciente precisa de dieta assistida [...] tem muito pouco enfermeiro, o enfermeiro não dá conta suprir tudo sabe [...]. (E02)

[...] falta muito profissional em todos os horários, se tivesse uma equipe completa em todos os horários, talvez a integração melhor entre diagnóstico e conduta seria mais eficaz [...] às vezes, eu preciso de um médico e não tenho um médico agora no horário que preciso, e como que eu vou comentar uma evolução ou uma regressão de um paciente. (E10) 
Evidencia-se que, apesar dos esforços dos profissionais para interação e articulação com os demais no cotidiano de trabalho, na unidade de Neurologia, a composição incompleta da equipe de saúde dificulta o trabalho em equipe. Os participantes reconhecem a necessidade do quantitativo adequado de profissionais e de ajuda mútua entre os agentes, na busca pela concretização da finalidade do trabalho - a integralidade da assistência. Porém, reforçam que o número insuficiente de profissionais inviabiliza o trabalho articulado e compromete o atendimento ao paciente.

[...] hoje em dia é exatamente, às vezes, a restrição que infelizmente a gente tem que ter de pessoal, é o fator principal. Eu acho que apesar de a gente fazer o trabalho multidisciplinar, o número de profissionais que a gente trabalha ainda é insuficiente para fazer um trabalho melhor [...]. (E43)

[...] acho que todos têm que está comprometido, a equipe não pode faltar, falta um já fica difícil para os outros. Quando precisa de ajuda, a colega se dispõe, a gente tem que pesar não só o nosso lado, mas o lado do colega também [...] comprometimento com o colega [...]. (E20)

A segunda categoria temática, Instrumentos não materiais do processo de trabalho na Neurologia, revela que os entrevistados percebem que a colaboração, a comunicação/diálogo, o auxílio mútuo, o relacionamento interpessoal e o objetivo compartilhado são elementos que influenciam o trabalho em equipe, os quais serão facilitadores ou dificultadores, a depender de como são vivenciados e desenvolvidos no contexto da Neurologia.

Os participantes relataram que as ferramentas de trabalho não palpáveis são essenciais para o desenvolvimento do trabalho em saúde, e inclusive, se bem utilizadas, facilitam o trabalho em equipe, por meio da ajuda mútua, da colaboração, do respeito, do sincronismo entre agentes e da comunicação adequada. Tais aspectos são percebidos como facilitadores para o trabalho em equipe e são ilustrados pelos depoimentos:

[...] aqui no setor tem uma dinâmica muito boa, com sincronismo entre as tarefas de cada profissão [...] sensibilidade da própria equipe. Quando um colega tá com problema, a equipe compactua com isso, em tá ajudando, em tá facilitando para que ele melhore [...]. (E1)

[...] respeito, entender melhor o colega [...] saber escutar o colega, ver a dificuldade dele e procurar a ajudar, e não criticar [...] de repente esse paciente tem piora no quadro, se o paciente não é seu, é do colega você tem que ajudar, às vezes, o colega não tem a prática ali na parada [parada cardiorrespiratória]. Na urgência, você tem que ajudar no que for preciso e através disso se torna uma equipe melhor [...]. (E19)

[...] eu acredito muito no trabalho em equipe. É um modelo de trabalho em que vários agentes eles estão imbuídos numa missão comum, apesar de realizar atividades distintas [...] você percebe que aquelas equipes que se comunicam melhor são equipes mais coesas, trabalham de uma forma mais dinâmica, o trabalho rende, o trabalho a gente vê resultados positivos [...]. (E23)

Os profissionais trouxeram à tona, em seus relatos, que a complementaridade de saberes, a troca e a articulação entre agentes envolvidos no processo de trabalho, são elementos facilitadores para o trabalho em equipe, na neurologia, e contribuem para melhor qualidade da assistência prestada. 
[trabalho em equipe] [...] eu entendo como sendo um trabalho centrado no paciente, onde cada profissional coloca a sua experiência (...)onde cada profissional pode dar a sua contribuição para realização daquele trabalho de forma mais multidisciplinar [...]. (E31)

[...] trabalho em equipe é vários profissionais, inclusive de outras áreas para poder complementar o trabalho um do outro, uma vez que não é possivel um profissional dominar todas as áreas do conhecimento. Então, a pessoa precisa de outros profissionais para interagir para tomar a melhor decisão, levando em consideração a opinião de todos [...]. (E44)

Por outro lado, os participantes chamam atenção para o fato de que a falta de iniciativa, a baixa colaboração, a pouca ajuda mútua, a comunicação ineficiente e o limitado trabalho coletivo representam dificultadores para o trabalho em equipe, conforme ilustram as falas a seguir:

[...] quando a pessoa não tem iniciativa de vê o que o paciente tá precisando e já ir lá e procurar e fazer [...] dificulta muito o trabalho em equipe, quando a pessoa não tem essa iniciativa [...]. (E13)

[...] a questão do próprio profissional não se dispor, não entender que o trabalho em equipe pode ser importante, porque cada um dentro do seu quadrado acha que desenvolvendo a sua parte é o suficiente, a gente sabe que não é [...] a falta de comunicação precisa melhorar muito [...]. (E22)

[...] os colegas se acabando de trabalhar, sendo que eu não tomo a iniciativa de trabalhar junto com o colega [...] tem que levar um paciente para exame e os outros colegas estão ocupados [...] você pode ir e você não toma iniciativa, você não faz e fica sentada [...] é um dos fatores que pode atrapalhar também [...]. (E14)

Para alguns participantes, o médico é um dos agentes que trabalha de maneira não colaborativa e se posiciona à margem da equipe, como se o trabalho dele fosse independente dos demais. Estabelece, com os outros agentes, uma relação interprofissional verticalizada, o que prejudica o trabalho coletivo e dificulta o trabalho em equipe. Isso fica registrado nos relatos:

[...] às vezes a gente percebe a parte médica tem um pouco de resistência a esse trabalho em equipe, querendo impor, e acho que a questão não é imposição, questão é discussão [...]. (E15)

[...] os médicos [...] ainda é a figura ainda mais difícil de ser atingida quando a gente fala em trabalho em equipe, porque eles entendem que o trabalho deles é dissociado dessa equipe, eles realizam o trabalho deles e aí o deles está finalizado, e a gente sabe que não é assim [...]. (E22)

A terceira categoria temática, Gerência: coordenação e liderança, revela percepções dos profissionais quanto à importância da gerência e da liderança na organização do serviço como elementos que influenciam na construção do trabalho em equipe. Os entrevistados enfatizaram que a gerência participativa, dialógica e a liderança são facilitadores para o trabalho integrado e em equipe. 
[...] acho que tem que ter uma pessoa presente que coordene todo mundo, um cabeça para mandar, "vamos reunir, vamos argumentar e todos têm que chegar a uma conclusão", sai um bom diagnóstico, um bom tratamento e uma boa resolução para aquele cliente ou paciente. (E10)

[...] eu acredito que o trabalho em equipe necessita de uma liderança informal, alguém ali do grupo no sentido de ser uma referência [...] começar a pegar essas pessoas para trocar experiências, para discutir melhor [...]. (E23)

[...] primeiro eu acho que a consciência da chefia [...] eu acho que quem tá no comando aqui da neurologia, eles têm muita essa visão de interdisciplinaridade, eles são abertos a discussão, querem saber quais as dificuldades [...] isso facilita bastante [trabalho em equipe] [...]. (E27)

Ainda no que tange à coordenação do trabalho, constata-se que, apesar de os profissionais do setor trabalharem juntos há bastante tempo, isso não garante articulação e trabalho coeso. É preciso que a gerência atue como elemento que promova o trabalho coletivo, caso contrário, se o profissional que coordena os agentes não tem essa percepção, isso dificultará o trabalho em equipe.

[...] são profissionais que trabalham há muito tempo juntos, mas não tem uma sintonia entre eles, é muito fragmentado o trabalho, são inclusive equipes mais difíceis de serem gerenciadas pelo profissional, só que a equipe é o espelho do profissional que está à frente dela [...] uma liderança negativa, a tendência é de o grupo trabalhar cada vez pior [...]. (E23)

\section{Discussão}

As entrevistas revelaram a presença de alguns elementos do processo de trabalho nos depoimentos dos participantes, como a finalidade, os agentes e os instrumentos não materiais (Gonçalves, 1992).

Quanto ao elemento agentes do processo de trabalho e trabalho em equipe, o número insuficiente de profissionais, dimensionamento inadequado e, aspectos macro-organizacionais do ambiente, como dificuldades de acessar outros profissionais, são fatores dificultadores na construção de uma equipe integrada, pautada na colaboração interprofissional (Walton et al., 2020; Ervin et al., 2018). A falta de profissionais restringe oportunidades para comunicação formal/informal e geram barreiras (Dariel \& Cristofalo, 2018), visto que a interação entre eles está diretamente ligada à capacidade de comunicar-se.

Isso inclusive compromete o desempenho da conclusão de metas e objetivos (Baird et al., 2019; Kendall-Gallagher et al., 2017), o que pode impactar na assistência prestada ao paciente em estado crítico que depende ainda mais da comunicação interprofissional efetiva para a solução rápida de problemas (Kendall-Gallagher et al., 2017). Desta forma, a comunicação, na perspectiva de troca e partilha, revela-se como potente ferramenta para viabilizar e fortalecer essa interação dos agentes (Baird et al., 2019), com vistas ao trabalho em equipe.

$\mathrm{Na}$ perspectiva dos instrumentos não materiais do processo de trabalho, neste estudo, chama atenção a clareza com que profissionais percebem e expressam que os agentes possuem atribuições distintas, mas isso não comprometerá o trabalho em equipe. Pois a colaboração é desenvolvida com o propósito de articular ações de várias especialidades, com vistas à atenção integral (Fox et al., 2017; van Dongen et al., 2016). Pode-se concretizar por meio do engajamento de profissionais que pensam em somar esforços, ou seja, trabalhar coletivamente de forma a produzir saúde com excelência (Peduzzi \& Agreli, 2018; Peruzzo et al., 2018). Neste sentido, os instrumentos não materiais do processo de trabalho viabilizam ações pautadas na lógica 
colaborativa e na integração entre as diversas áreas e saberes (Peduzzi \& Agreli, 2018; Barros et al., 2018; Escalda \& Parreira, 2018) além disso, é uma potente ferramenta facilitadora para o trabalho em equipe.

Por outro lado, baixa colaboração e limitado trabalho coletivo dificultam trabalho em equipe (Rosen et al., 2018; van Dogen et al., 2016; Carney et al., 2019). Algumas vezes, o médico é um profissional que trabalha isoladamente e de maneira não colaborativa, de difícil acesso para estabelecer a comunicação com outras categorias profissionais, posicionando-se à margem da equipe (Gleddie et al., 2018). Isto fragmenta o trabalho e prejudica a finalidade do processo de trabalho.

Contudo, o estabelecimento da confiança, respeito, conhecimento e experiência, são apontados como os principais fatores que facilitam as relações de trabalho eficazes com outros profissionais (Gleddie et al., 2018; Rydenfält et al., 2019) possibilitam atuações profissionais mais criativas e favorecem a comunicação adequada (Golom \& Schreck, 2018; Peduzzi \& Agreli, 2018; van Dongen et al., 2016; Escalda \& Parreira, 2018). Isso sinaliza estratégias a serem implementas no contexto da unidade de Neurologia.

O gerenciamento adequado impacta diretamente na organização do serviço para os profissionais da unidade supracitada. O gerente ou líder quando é um profissional que tem a compreensão da importância da coletividade e do trabalho integrado, desperta isso nos outros agentes e promove o trabalho em equipe. Esses achados convergem com a literatura, a qual afirma que incentivar a participação pode colaborar para o consenso entre os seguidores e envolvê-los na construção de uma visão compartilhada, pois a organização é vista como interligada e interdependente (Thiagarajan \& McKimm, 2019).

As funções gerenciais fundamentais para o serviço são permeadas de características relativas à liderança, à coordenação e a métodos de comunicação eficientes. Além disso, as estruturas de gerenciamento precisam ser explicitamente colaborativas, apoiar o desenvolvimento e o processo da equipe e fornecer feedback regularmente sobre o desempenho da equipe, isso viabiliza para que os membros da equipe alcancem os objetivos (Ferreira et al., 2019; Fiscella et al., 2017), sendo fundamental na prevenção de eventos adversos na assistência em saúde (Schwartz et al., 2018) além de favorecer a segurança do paciente (Im \& Aaronson, 2020).

A concepção de liderança não necessariamente está vinculada à hierarquia ou cargo, mas sim a agentes da equipe que sejam reconhecidos como referência e que saibam conduzir os profissionais na construção do trabalho em equipe. Além disso, liderança consistente e clara, possibilita aos profissionais da equipe lidarem melhor com os desafios que eles experimentam enquanto trabalham, e garante que as equipes estejam suficientemente preparadas para realizar o trabalho necessário (Baird et al., 2019; Franz et al., 2018; Whitehair et al., 2018), especialmente em um cenário no qual os pacientes demandam assistência contínua e crítica como o da Neurologia.

Ressalta-se que a centralidade do processo de trabalho nessa unidade de Neurologia investigada, no que tangencia o trabalho em equipe, está focada, especialmente, nos instrumentos não materiais do processo de trabalho que se revelam, inclusive, quando os profissionais trazem à tona temáticas como os agentes do processo de trabalho e a gerência. Isso evidencia estreita proximidade dos achados com os aspectos relacionais.

$\mathrm{Na}$ concepção do projeto, pretendia-se desenvolver rodas de discussão com os agentes da referida unidade, com base nos achados do estudo para disparar a discussão e a reflexão dos resultados, com intuito de ressignificar o trabalho em equipe, naquele contexto. Entretanto, isso não foi possível devido às condições de trabalho inviabilizarem a ausência dos profissionais da unidade. Desta forma, procedeu-se à devolutiva dos resultados aos profissionais da Neurologia e banner contemplando os resultados da pesquisa, bem como apresentação e discussão dos resultados nos três turnos de trabalho, na unidade.

Os limites deste estudo referem-se à sua realização em uma única unidade de Neurologia. Entretanto, ainda que isso pese como limitação, não se tem a pretensão de generalizar os achados. Além disso, acredita-se que o cotidiano, a vivência e a dinâmica do trabalho encontrados no referido contexto se assemelhem a vários outros serviços de saúde. 


\section{Considerações Finais}

Utilizando-se da análise temática, esta investigação possibilitou identificar as percepções dos profissionais de saúde sobre o trabalho em equipe, em uma unidade de Neurologia, segundo os agentes da equipe de saúde.

A complementaridade, a troca de saberes e a articulação efetiva viabilizam o trabalho em equipe e contribuem para a qualidade da assistência prestada. Inclusive, elementos não materiais, como colaboração, ajuda mútua e comunicação, são identificados como fundamentais para a realização do trabalho em equipe. Nessa perspectiva, a compreensão, por parte do gerente ou líder, da importância da coletividade e do trabalho integrado, promove esse entendimento e favorece a valorização pelos demais agentes.

Por outro lado, a composição incompleta da equipe e as relações interprofissionais ancoradas na baixa colaboração e comunicação dificultam o trabalho em equipe, prejudicam a articulação entre saberes e fazeres, e geram prejuízos para o alcance da finalidade do processo de trabalho em saúde, por mais que os agentes se esforcem para a realização de trabalho integrado e articulado.

Chama a atenção o fato de os profissionais destacarem o papel do líder da equipe como potente elemento na construção do trabalho em equipe, o que revela que a concepção de liderança diz respeito a agentes da equipe que são reconhecidos como referência. Isso evidencia a relevância da formação de profissionais na área da saúde que saibam liderar equipes e mobilizar agentes. Outro aspecto a ser destacado refere-se à gerência como valiosa ferramenta para sensibilizar e possibilitar envolvimento e participação dos agentes no trabalho, contribuindo para o trabalho em equipe. Espera-se que os resultados deste estudo forneçam subsídios para que a gestão se aproprie das condições, necessidades e vivência dos profissionais da referida unidade, com vistas à melhoria da dinâmica do trabalho.

Não se pretende esgotar a temática, mas enfatiza-se que o estudo desvelou a necessidade de que a liderança de equipe e as relações interprofissionais sejam fundamentadas na comunicação efetiva, no trabalho em equipe e em tomadas de decisões compartilhadas. Aposta-se na realização de novas pesquisas utilizando-se a Técnica do Incidente Crítico para evidenciar situações reais a respeito de trabalho em equipe, na unidade de Neurologia, e identificar comportamentos que favoreçam e dificultam tal modalidade de trabalho para se pensar em estratégias em serviço com vistas ao fortalecimento dos aspectos positivos e superação das fragilidades.

\section{Referências}

Arruda, P. L., Xavier, R. O., Lira, G. G., Arruda, R. G., Melo, R. A., \& Fernandes, F. E. C. V. (2019). Clinical evolution and survival of neurocritical patients. Revista da Escola de Enfermagem da USP, 53, e03505.

Baird, J., Ashland, M., \& Rosenbluth, G. (2019). Interprofessional teams current trends and future directions. Pediatric Clinics of North America, 66(4,) 739750 .

Barros, N. F., Spadacio, C., \& Costa, M. V. (2018). Interprofessional work in Integrative and Complementary Practices in the context of Primary Health Care: potentials and challenges. Saúde em Debate, 42(Spe 1), 163-173.

Bragadóttir, H., Kalisch, B. J., \& Tryggvadóttir, G. B. (2017). Correlates and predictors of missed nursing care in hospitals. Journal of Clinical Nursing, 26(11-12), 1524-1534.

Carney, P. A., Thayer, E. K., Palmer, R., Galper, A. B., Zierler, B., \& Eiff, M. P. (2019). The benefits of interprofessional learning and teamwork in primary care ambulatory training settings. Journal of Interprofessional Education \& Practice, 15, 119-126.

Dariel, O. P. D., \& Cristofalo, P. (2018). A meta-ethnographic review of interprofessional teamwork in hospitals: what it is and why it doesn't happen more often. Journal of Health Services Research \& Policy, 23(4), 272-279.

Ervin, J. N., Kahn, J. M., Cohen, T. R., \& Weingart, L. R. (2018). Teamwork in the Intensive Care Unit. The American Psychologist, 73(4), $468-477$.

Escalda, P., \& Parreira, C. M. S. F. (2018). Dimensions of interprofessional work and of collaborative practices developed at a primary care unit by a Family Health team. Interface - Comunicação, Saúde e Educação, 22(Suppl 2), 1717-1727.

Ferreira, V. H. S., Teixeira, V. M., Giacomini, M. A., Alves, L. R., Gleriano, J. S., \& Chaves, L. D. P. (2019). Contributions and challenges of hospital nursing management: scientific evidence. Revista Gaúcha de Enfermagem, 40, e20180291. 
Fiscella, K., Mauksch, L., Bodenheimer, T., \& Salas, E. (2017). Improving Care Teams Functioning Recommendations from Team Science. The Joint Commission Journal on Quality and Patient Safety, 43(7), 361-368.

Fox, L., Onders, R., Hermansen-Kobulnicky, C. J., Nguyen, T., Myran, L., Linn, B., \& Hornecker, J. (2017). Teaching interprofessional teamwork skills to health professional students: a scoping review. Journal of Interprofessional Care, 32(2), 127-135.

Franz, S., Muser, J., Thielhorn, U., Wallesch, C. W., \& Behrens, J. (2018). Inter-professional communication and interaction in the neurological rehabilitation team: a literature review. Disability and Rehabilility, 42(11), 1607-1615.

Gleddie, M., Stahlke, S., \& Paul, P. (2018). Nurses' perceptions of the dynamics and impacts of teamwork with physicians in labour and delivery. Journal of Interprofessional Care, 01-11.

Golom, F. D., Schreck, J. S. (2018). The journey to interprofessional collaborative practice are we there yet?. Pediatric Clinics of North America, 64(1), 1-12.

Gonçalves, R. B. M. (1992). Práticas de saúde: processos de trabalho e necessidades. CEFOR.

Im, D., \& Aaronson, E. (2020). Best Practices in Patient Safety and Communication. Emergency Medicine Clinics of North America, 38(3), 693-703.

Kendall-Gallagher, D., Reeves, S., Alexanian, J. A., \& Kitto, S. (2017). A nursing perspective of interprofessional work in critical care: findings from a secondary analysis. Journal of Critical Care, 38, 20-26.

Minayo, M. C. S., Deslandes, S. F., \& Gomes, R. (2016). Pesquisa social: teoria, método e criatividade. Petrópolis: Vozes.

Peduzzi, M., \& Agreli, H. F. (2018). Teamwork and collaborative practice in Primary Health Care. Interface - Comunicação, Saúde e Educação, 22(Suppl 2), $1525-1534$

Peduzzi, M., Agreli, H. L. F., Silva, J. A. M., \& Souza, H. S. (2020). Teamwork: revisiting the concept and its developments in inter-professional work. Trabalho, Educação e Saúde, 18(Suppl 1), e0024678.

Peduzzi, M., Aguiar, C., Lima, A. M. V., Montanari, P. M., Leonello, V. M., \& Oliveira, M. R. (2019). Expansion of the interprofessional clinical practice of Primary Care nurses. Revista Brasileira de Enfermagem, 72(Suppl 1), 114-121.

Peruzzo, H. E., Bega, A. G., Lopes, A. P. A. T., Haddad, M. C. F. L., Peres, A. M., \& Marcon, S. S. (2018). The challenges of teamwork in the family health strategy. Escola Anna Nery Revista de Enfermagem, 22(4), 1-9.

Purvis, T. L., \& Saylor, D. (2019). Burnout and resilience among neurosciences critical care unit staff. Neurocritical Care, 31(2), 406-410.

Rosen, M. A., Diaz Granados, D., Dietz, A. S., Benishek, L. E., Thompson, D., Pronovost, P. J., \& Weaver, S. (2018). Teamwork in healthcare: key discoveries enabling safer, high-quality care. The American Psychologist, 73(4), 433-450.

Rydenfält, C., Borell, J., \& Erlingsdottir, G. (2019). What do doctors mean when they talk about teamwork? possible implications for interprofessional care. Journal of Interprofessional Care, 33(6), 714-723.

Schwartz, M. E., Welsh, D. E., Paull, D. E., Knowles, R. S., DeLeeuw, L. D., Hemphill, R. R., Essen, K. W., \& Sculli, G. (2018). The effects of crewresource management on teamworkandsafetyclimateatVeterans Health Administration facilities. Journal of Healthcare Risk Management, 38(1), 17-37.

Thiagarajan, P., \& McKimm, J. (2019). Mapping transactional analysis to clinical leadership models. British Journal of Hospital Medicine, 80(10), 600-604.

Tong, A., Sainsbury, P., \& Craig, J. (2007). Consolidated criteria for reporting qualitative research (COREQ): a 32 -item checklist for interviews and focus groups. International Journal for Quality in Health Care, 19(6), 349-357.

van Dongen, J. J. J., Lenzen, S. A., Bokhoven, M. A., Daniels, R., Weijden, T. V. D., \& Beurskens, A. (2016). Interprofessional colaboration regarding patientes care plans in primary care: a focus grup study into influential factors. BMC Family Practice, 17, 58.

Walton, V., Hodgen, A., Long, J. C., Johnson, J., \& Greenfield, D. (2020). Exploring interdisciplinary team work to support effective ward rounds. International Journal for Quality in Health Care Assurance, 33(4-5), 373-387.

Whitehair, L., Hurley, J., \& Provost, S. Envisioning successful teamwork: An exploratory qualitative study of team processes used by nursing teams in a paediatric hospital unit. Journal of Clinical Nursing, 27(23-24), 4257-4269. 